EESTI NSV TEADUSTE AKADEEMIA TOIMETISED. XV KÖIDE FUUSIKA-MATEMAATIKA- JA TEHNIKATEADUSTE SEERIA. 1966, NR. 3

ИЗВЕСТИЯ АКАДЕМИИ НАУК ЭСТОНСКОЙ ССР. ТОМ ХV СЕРИЯ ФИЗИКО-МАТЕМАТИЧЕСКИХ И ТЕХНИЧЕСКИХ НАУК. 1966, № 3

И. МАУЭР

\title{
ПРИБЛИЖЕННЫЙ МЕТОД РЕШЕНИЯ ЗАДАЧИ ВЫПУКЛОГО ПРОГРАММИРОВАНИЯ
}

Задача выпуклого программирования заключается в минимизации выпуклого функционала $f(x)$ прн выпуклых ограничениях $g_{i}(x) \leqslant 0(i=1, \ldots, m)$, где $x \in E^{n}$. В последние годы разработан целый ряд методов для решения такой задачи. По характеру и времени возникновения их можно группировать на методы лагранжевые, прямые. погружные. В последней группе известны методы [1-4]. Предлагаемый в данной работе метод является видоизменением хорошо известного метода погружения [1].

Рассмотрим задачу выпуклого программирования с многими ограничениями: минимизировать

$$
f(x)
$$

при ограничениях

$$
g_{i}(x) \leqslant 0 \quad(i=1, \ldots, m) .
$$

Предположим, что $f(x), g_{i}(x)(i=1, \ldots, m)$ - два раза непрерывно дифференцируемые функционалы и что область $D$, определенная ограничениями задачи, непустая и ограниченная.

Приближенное решение задачи (1), (2) сводим к решению ряда задач $r=1, \ldots, r^{\prime}$ с одним ограничением: минимизировать

$$
f(x)
$$

при ограничении

$$
\frac{1}{2} \sum_{i=1}^{m} S\left(g_{i}(x)\right)\left(g_{i}(x)\right)^{2}-\Delta_{r} \leqslant 0
$$

где

$$
S(t)=\left\{\begin{array}{l}
1, \text { если } t>0 \\
0, \text { если } t \leqslant 0
\end{array}\right.
$$

и $\Delta_{r}-$ положительное число. Эти задачи отличаются только по значению $\Delta_{r}$. Как легко доказывается, ограничение (4) представляет собой выпуклый и непрерывно дифференцируемый функционал. А область $D_{\Delta_{\tau}}$, определенная ограничением (4), содержит очевидно собственно внутреннюю точку.

$$
\text { Чнсла } \Delta_{r}\left(r=1, \ldots, r^{\prime}\right) \text { выбираем убывающими } \Delta_{1}>\Delta_{2}>\ldots>\Delta_{r} \text {. }
$$




\section{О бо з н чения}

Используем в дальнейшем следующие обозначения:

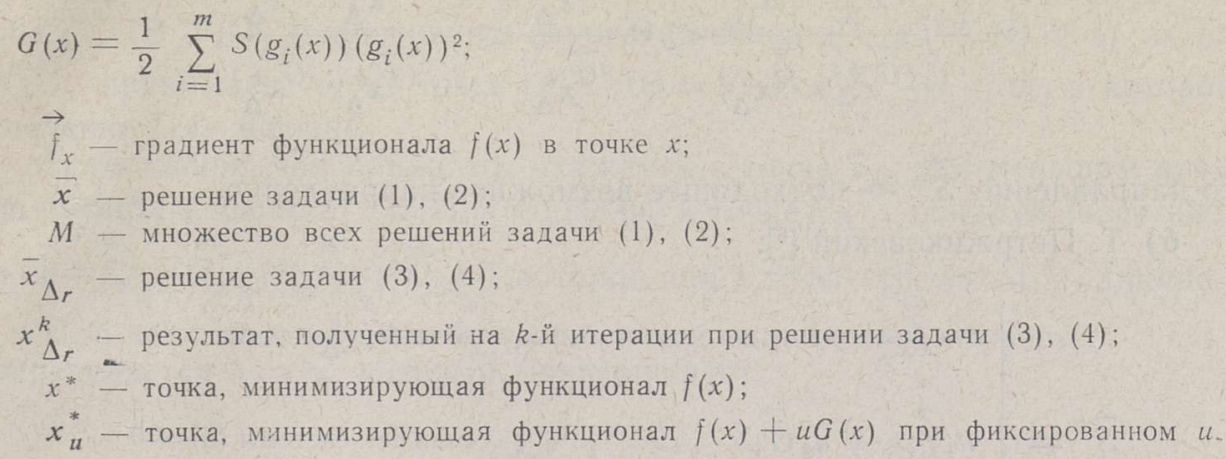

\section{О решении задачи (3), (4)}

Решаем задачу (3), (4) с определенным значением $\Delta$.

За исходную точку решения выбираем какую-нибудь точку $x_{\Delta}^{0} \in D_{\Delta}$ и после $\kappa$-й итерации получим $x_{\Delta}^{k}$. Последующую точку $x_{\Delta}^{k+1}$ вычисляем по формуле

$$
x_{\Delta}^{k+1}=x_{\Delta}^{k}+\lambda_{k} \vec{S}^{k}
$$

где направление движения $\vec{S}^{k}$ и скаляр $\lambda_{k}$ определяем таким образом, что в результате вычислений получим последовательность $\left\{x^{k} \Delta\right\}$ такую, что

$$
\lim _{k \rightarrow \infty} x_{\Delta}^{k}=\bar{x}_{\Delta}
$$

Есть разные возможности определения вектора $\vec{S}{ }^{k}$ и скаляра $\lambda_{k}$. В данном случае считаем целесообразным воспользоваться такими, которые задают движение в пределах области $D_{\Delta}$ и в общем различно определяют направление в краевых точках области, - методами возможных направлений [5-8]. В близких друг от друга краевых точках области $D_{\Delta}$ градиент функционала $G(x)$ может резко отличаться по направлениям и вызывать «зигзагообразное» движение при применении отмеченных методов. Поэтому введем число $\Theta>0$, известное из работы Г. Зойтендейка $\left[{ }^{7}\right]$, и приведем способы определения направления по методу $\left[{ }^{7}\right]$ и по методам $\left[{ }^{5,6}\right]$, несколько модифицировав последние.

a) M. Хансон $\left.{ }^{[5}\right]$ :

$$
\vec{S}^{k}=\left\{\begin{array}{l}
-\vec{f}_{x_{\Delta}^{k}} \\
-\vec{f}_{x_{\Delta}^{k}}-\Theta \frac{\left|\vec{f}_{x_{\Delta}^{k}}\right|}{\left|\vec{G}_{x_{\Delta}^{k}}\right|} \vec{G}_{x_{\Delta}^{k}}
\end{array} \quad, \quad \text { если } G\left(x_{\Delta}^{k}\right)<\Delta,\right.
$$


Пользуясь только непрерывной дифференцируемостью функционалов $f(x)$ и $G(x)$, можно показать, что если

$$
-\frac{\left(\vec{f}_{x_{\Delta}^{k}}, \vec{G}_{x_{\Delta}^{k}}\right)}{\left(\vec{G}_{x_{\Delta}^{k}}, \vec{G}_{x_{\Delta}^{k}}\right)}<\theta \frac{\left|\vec{f}_{x_{\Delta}^{k}}\right|}{\left|\vec{G}_{x_{\Delta}^{k}}\right|}<-\frac{\left(\vec{f}_{x_{\Delta}^{k}}, \vec{f}_{x_{\Delta}^{k}}\right)}{\left(\vec{f}_{x_{\Delta}^{k}}, \vec{G}_{x_{\Delta}^{k}}\right)}
$$

то направление $\vec{S}^{k}$ - подходящее возможное направление.

б) Т. Петржиковский [ $\left.{ }^{6}\right]$ :

$$
\vec{S}^{k}=\left\{\begin{array}{l}
-\vec{f}_{x_{\Delta}^{k}}^{k} \\
u_{1} \vec{f}_{x_{\Delta}^{k}}+u_{2} \vec{G}_{x_{\Delta}^{k}} \\
0
\end{array}\right.
$$

, если $G\left(x_{\Delta}^{k}\right)<\Delta$

, если det. системы $(5) \neq 0$ и $G\left(x_{\Delta}^{k}\right)=\Delta$,

, если det. системы $(5)=0$,

где $u_{1}$ и $u_{2}$ определяются как решение системы линейных уравнений

$$
\begin{aligned}
& u_{1}\left(\vec{f}_{x_{\Delta}^{k}}, \vec{f}_{x_{\Lambda}^{k}}\right)+u_{2}\left(\vec{G}_{x_{\Delta}^{k}}, \vec{f}_{x_{\Delta}^{k}}\right)=-1, \\
& u_{1}\left(\vec{f}_{x_{\Delta}^{k}}, \vec{G}_{x_{\Delta}^{k}}\right)+u_{2}\left(\vec{G}_{x_{\Delta}^{k}}, \vec{G}_{x_{\Delta}^{k}}\right)=-\Theta .
\end{aligned}
$$

в) Г. Зойтендейк $\left[{ }^{7}\right]$ :

$$
\vec{S}^{k}=\left\{\begin{array}{cc}
-\vec{f}_{x_{\Delta}^{k}} & , \text { если } G\left(x_{\Delta}^{k}\right)<\Delta \\
y^{k} & , \text { если } G\left(x_{\Delta}^{k}\right)=\Delta
\end{array}\right.
$$

где $y^{k}$ определяется как решение следующей задачи программирования: максимизировать б при ограничениях

$$
\begin{aligned}
& \vec{f}_{x_{\Delta}^{k}} y^{k} \leqslant-\sigma \\
& \vec{G}_{x_{\Delta}^{k}} y^{k} \leqslant-\Theta \sigma \\
& \left|y^{k}\right| \leqslant C \text { (некоторая константа). }
\end{aligned}
$$

Легко сообразить, что в зависимости от значения $\Theta$ определяемое направление образует больший или меньший угол с касательной гиперплоскостью функционала $G(x)$ в точке $x_{\Delta}^{k}$, и в зависимости от задачи целесообразно в вычислениях выбрать $\Theta$ то больше, то меньше единицы.

Выберем достаточно малое положительное число $\varepsilon>0$, при помощи которого обнаруживаем движение с малым шагом. Если $\lambda_{k}\left|\vec{S}^{k}\right|>\varepsilon$ 
$\left(\vec{S}^{k}\right.$ огределено $\left.\Theta=1\right)$, то продолжаем вычисления с $\Theta=1$. В противном случае наряду с $\lambda_{\Delta}^{k+1}(\Theta=1)\left(x_{\Delta}^{k+1}\right.$ определено $\left.\Theta=1\right)$ вычисляем еще $x_{\Delta}^{k+1}(\Theta>1)$ и, если $f\left(x_{\Delta}^{k+1}(\Theta=1)\right)>f\left(x_{\Delta}^{k+1}(\Theta>1)\right)$, то $x_{\Delta}^{k+1}=$ $=x_{\Delta}^{k+1}(\Theta>1)$. В противном случае вычисляем и $x_{\Delta}^{k+1}(\Theta<1)$ и за $x_{\Delta}^{k+1}$ принимаем ту из точек $x_{\Delta}^{k+1}(\Theta=1)$ и $x_{\Delta}^{k+1}(\Theta<1)$, в которой значение $f(x)$ меньше.

Естественно, чем ближе мы окажемся к точке $\bar{x}_{\Delta}$, тем меньшим нужно выбирать число $\varepsilon>0$. Приближение к точке $\bar{x}_{\Delta}$ обнаруживает число $\left(-\vec{f}_{x_{\Delta}^{k}}, \vec{G}_{x_{\Delta}^{k}}\right) /\left(\left|\vec{f}_{x_{\Delta}^{k}}\right|\left|\vec{G}_{x_{\Delta}^{k}}\right|\right)$,которое при $k \rightarrow \infty$ стремится к единице.

При всех этих методах $\lambda_{k}$ вычисляется одинаковым образом: определяется $\lambda=\lambda_{k}$, который минимизирует

$$
f\left(x_{\Delta}^{k}+\lambda \vec{S}^{k}\right)
$$

при ограничении

$$
G\left(x_{\Delta}^{k}+\lambda \overrightarrow{S^{k}}\right)-\Delta \leqslant 0
$$

Нетрудно проверить, что для методов а), б) $\vec{S}^{k}=0$, а для метода в) $\rightarrow$ $x_{\Delta}^{k}=0$ или $\max \sigma=0$ являются необходимыми и достаточными условиями для того, чтобы $x_{\Delta}^{k}=\bar{x}_{\Delta}$.

\section{Сходимость метода}

Tе o p e м a

Соответственно любому числу $\varepsilon>0$ найдется такое число $\Delta_{0}>0$, что если $0<\Delta<\Delta_{0}$, то $\mathrm{Q}\left(\bar{x}_{\Delta}, M\right)<\varepsilon$.

Доказательство. Если $x^{*} \in D$, то $x^{*}=\bar{x}=\bar{x}_{\triangle}$ и при всех $0<$ $<\Delta<\infty$ Q $\left(\bar{\Lambda}_{\Delta}, M\right)=0$

Предположим, что $x^{*} \notin D$.

Воспользуемся теоремой, которая очевидно равносильна известной теореме Т. Петржиковского [ $\left.{ }^{1}\right]$ : соответственно выбранному числу $\varepsilon>0$ найдется такое число $u_{0}>0$, что если $u_{0}<u<\infty$, то $\varrho\left(x_{u}^{*}, M\right)<\varepsilon$.

Любому $\bar{u}>0$ и соответствуюшему $x_{\bar{u}}^{*}$ можно сопоставить $\Delta>0$ и соответствующее $\bar{x}_{\Delta}$ таким образом, что $x_{\bar{u}}^{*}=\bar{x}_{\Delta \text { и }} \Delta=G\left(x_{\bar{u}}^{*}\right)$. Действительно, точка $x_{\bar{u}}^{*}$, минимизирующая функционал $f(x)+\vec{u} G(x)$, минимизирует и функционал $f(x)+\bar{u}\left(G(x)-G\left(x_{\bar{u}}^{*}\right)\right)$, а, следовательно, $\left(x_{u}^{*}, \bar{u}\right)$ является седловой точкой функционала Лагранжа $f(x)+u(G(x)-\Delta)$ задачи (3), (4) с $\Delta=G\left(x_{u}^{*}\right)$. По теореме Куна-Таккера $x_{\bar{u}}^{*}=\bar{x}_{\Delta}$, если $\Delta=G\left(x_{u}^{*}\right)$.

Таким образом, для любого $\Delta=G\left(x_{u}^{*}\right)$ с $u_{0}<u<\infty \varrho\left(\bar{x}_{\Delta}, M\right)<\varepsilon$.

Выберем $\Delta_{0}=G\left(x_{u_{0}}^{*}\right)$ и покажем, что для задачи (3), (4) с любым значением $0<\Delta<\Delta_{0}$ найдется такое $u>u_{0}$, что $\Delta=G\left(x_{u}^{*}\right)$. 
Пусть функционал Лагранжа задачи (3), (4) с $\Delta<\Delta_{0}$ имеет седловую точку $\left(x_{u}^{*}, u\right)$. Множитель $u>0$, так как $x^{*} \notin D_{\Delta}$ (очевидно $D_{\Delta} \subset D_{\Delta_{0}}$ $\left(\Delta<\Delta_{0}\right)$, а $\left.x^{*} \notin D_{\Delta_{0}}\left(u_{0}>0\right)\right)$. Следовательно, $\Delta=G\left(x_{u}^{*}\right)$. дение.

Для установления соотношения $u>u_{0}$ проведем следующее рассуж-

Так как $\vec{f}_{x_{u_{0}}^{*}}+u_{0} \vec{G}_{x_{u_{0}}^{*}}=0$ и $\vec{f}_{x_{u_{0}}^{*}}+u \vec{G}_{x_{u_{0}}^{*}} \neq 0 \quad\left(u \neq u_{0}\right)$, то

$$
f\left(x_{u}^{*}\right)+u\left(G\left(x_{u}^{*}\right)-G\left(x_{u_{0}}^{*}\right)\right)<f\left(x_{u_{0}}^{*}\right) .
$$

С другой стороны, по определению седловой точки

$$
f\left(x_{u}^{*}\right)+u_{0}\left(G\left(x_{u}^{*}\right)-G\left(x_{u_{0}}^{*}\right)\right) \geqslant f\left(x_{u_{0}}^{*}\right) .
$$

Соединяем полученные неравенства:

$$
\frac{f\left(x_{u_{0}}^{*}\right)-f\left(x_{u}^{*}\right)}{u_{0}} \leqslant \Delta-\Delta_{0}<\frac{f\left(x_{u_{0}}^{*}\right)-f\left(x_{u}^{*}\right)}{u} .
$$

Учитывая, что $u_{0}>0, u>0$ и что было предположено $\Delta<\Delta_{0}$, получим соотношение $u>u_{0}$. Теорема доказана.

Следствие. Если $\lim _{r \rightarrow \infty} \Delta_{r}=0$, то $\lim _{r \rightarrow \infty} \bar{x}_{\Delta_{r}}=\bar{x}$.

Действительно, по доказанной теореме соответственно выбранному числу $\varepsilon>0$ найдется ограниченное множество $N$, содержащее множество $M$ и все точки $\bar{x}_{\Delta_{r}}$, для которых $\Delta_{r}<\Delta_{0}$. Из-за компактности множества $N$ из последовательности $\left\{\bar{x}_{\Delta_{r}}\right\}\left(\Delta_{r}<\Delta_{0}\right)$ можно выделить сходящуюся подпоследовательность, которую для простоты отметим таким же образом $\left\{\bar{x}_{\Delta_{r}}\right\}, \lim _{r \rightarrow \infty} \bar{x}_{\Delta_{r}}=\underline{x}$. Предположим, что $\varrho(\underline{x}, M)=\varepsilon^{*}>0$ и выбираем $0<\varepsilon<\varepsilon^{*}$. По теореме найдется такое $\Delta_{0}$, что, если $0<\Delta_{r}<$ $<\Delta_{0}$, то $\mathrm{o}\left(\bar{x}_{\Delta_{r}}, M\right)<\varepsilon$ и, следовательно, $\varrho(x, M) \leqslant \varepsilon<\varepsilon^{*}$. Полученное противоречие доказывает, что $\underline{Q}(\underline{x}, M)=0$ и $\underline{x}=\bar{x}$.

Приведем числовой пример:

минимизировать

при ограничениях

$$
-2 x_{1}-x_{2}
$$

$$
\begin{array}{r}
5 x_{1}^{2}+2 x_{2}^{2}+6 x_{1} x_{2}-4 x_{1}-2 x_{2}-3 \leqslant 0 \\
3 x_{1}+2 x_{2}-2 \leq 0 \\
-x_{1}-x_{2} \leq 0 .
\end{array}
$$

Приближенное решение задачи найдем как решение следующей задачи: минимизировать

$$
-2 x_{1}-x_{2}
$$

при ограничении

$$
\begin{gathered}
S\left(5 x_{1}^{2}+2 x_{2}^{2}+6 x_{1} x_{2}-4 x_{1}-2 x_{2}-3\right)\left(5 x_{1}^{2}+2 x_{2}^{2}+6 x_{1} x_{2}-4 x_{1}-2 x_{2}-3\right)^{2}+ \\
+S\left(3 x_{1}+2 x_{2}-2\right)\left(3 x_{1}+2 x_{2}-2\right)^{2}+S\left(-x_{1}-x_{2}\right)\left(-x_{1}-x_{2}\right)^{2}-\Delta \leqslant 0 .
\end{gathered}
$$

Решения задачи $\bar{x}_{\Delta}=\left(\bar{x}_{1 \Delta}, \bar{x}_{2 \Delta}\right)$ при значениях $\Delta=\Delta_{r}(r=1, \ldots)$ приведены в нижеследующей таблице. 


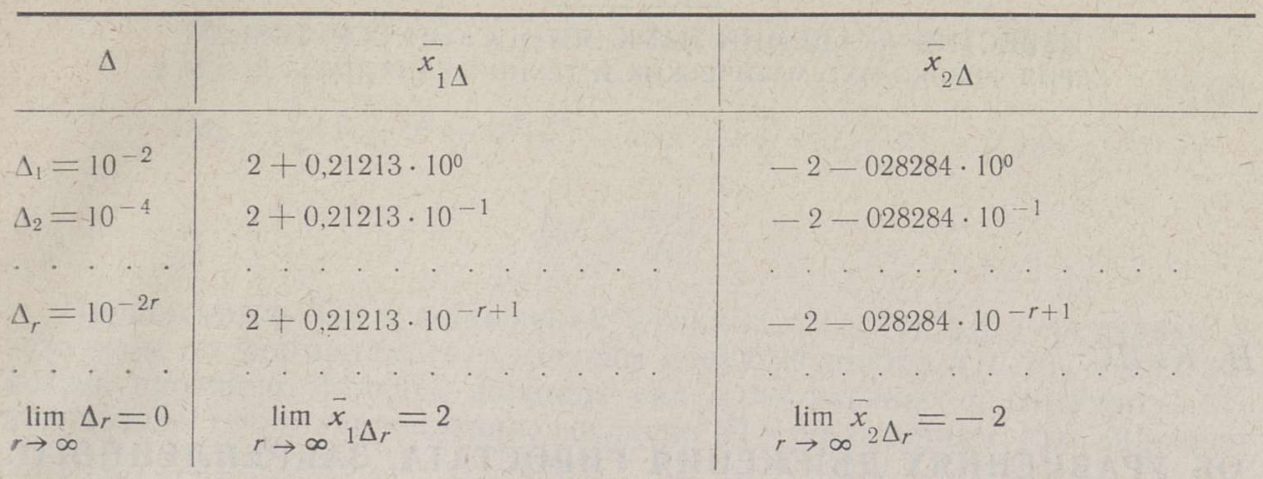

3 ам еч ани е 1. В практических вычислениях за исходную точку решения каждой новой задачи нужно выбирать точку приближенной проекции решения предыдущей задачи на область, определенную ограничением новой задачи.

3 а меч ание 2. Так как не имеется оценки для $\delta\left(\bar{x}_{\Delta}, M\right)$ или $f\left(\bar{x}_{\Delta}\right)-f(\bar{x})$, то требование точности решения можно задать с помощью некоторого вектора $\vec{\varepsilon}=$ $=\left(\varepsilon_{1}, \ldots, \varepsilon_{m}\right)>0: \bar{x}_{\Delta}$ считаем решением задачи с требуемой точностью, если

$$
g_{i}\left(\bar{x}_{\Delta}\right) \leqslant \varepsilon_{i} \quad(i=1, \ldots, m)
$$

для чего $\Delta$ должно быть меньше некоторого $\Delta_{0}$.

\section{Л И T E P A T Y P A}

1. Pi etrzykowski T., Inf. Process 1962, Amsterdam, 1963, pp. 185-189.

2. C a r r o 11 Ch. W., Oper. Res., 9, № 2, 169-185 (1961)

3. F i a c с о A. V., M c C or m i ck, Manag. Sci., 10, № 2, 360-366 (1964).

4. Бусленко Н. П., Соколов Г. А., Экономика и матем. методы, 1, вып. 1 , $123-136$ (1965).

5. H a n s o n M. A., Austral. J. Statist., 5, № 13, 14-19 (1963).

6. Pi etrzykowski. T., Prace zakl. apar. mat. PAN, A13, 1-22 (1961)

7. З ойтендейк Г., Методы возможных направлений, М., 1963.

8. Зуховицкий С. И., Поляк Р. А., Примак М. Е., Докл. АН СССР, 153. № 5, $991-994$ (1963).

Ннститут кибернетики

Академии наук Эстонской ССР
Поступила в редакцию $30 /$ XI 1965

\section{MAUER}

\section{LIGIKAUDNE MEETOD KUMERPROGRAMMEERIMISULESANDE LAHENDAMISEKS}

Paljude kitsendavate tingimustega kumerprogrammeerimisülesande ligikaudne lahendamine taandatakse mitme ühe kỗrvaltingimusega programmeerimisülesande lahendamisele.

\section{MAUER}

\section{AN APPROXIMATE METHOD FOR SOLVING CONVEX PROGRAMMING PROBLEMS}

The approximate solving of problems of convex programming with many constraints is reduced to solving a number of similar problems with a single one. 